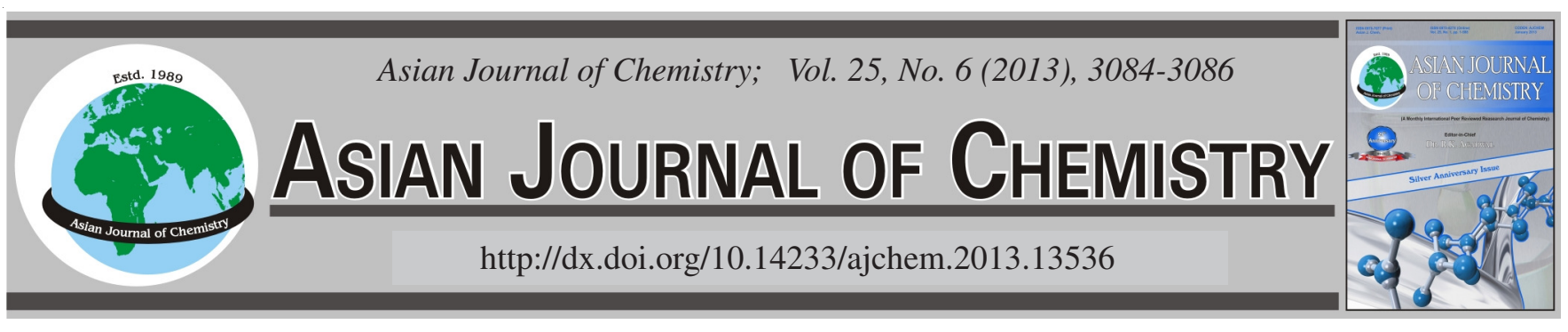

\title{
Hydrothermal Synthesis of Carbon Micro-Particles and Their Electrochemical Performance as Anode Materials for Lithium Ion Batteries
}

\author{
ZHIJUN YAN $^{*}$ and GANG ZHU
}

Department of Chemistry and Chemical Engineering, Xi'an University of Arts and Science, Xi'an, 710065, P.R. China

*Corresponding author: Fax: +86 29 88258550; Tel: +86 29 88258553; E-mail: zhijunyanxawl@ sina.com

Carbon micro-particles with smooth surface and sphere shape were prepared through a facile hydrothermal method using $\beta$-cyclodextrin as carbon source. Galvanostatic charge-discharge method was used to investigate the electrochemical performance of carbon microparticles. The results showed that carbon micro-particle anode exhibited an initial discharge capacity of $571 \mathrm{mAh} / \mathrm{g}$ and a reversible capacity above $345 \mathrm{mAh} / \mathrm{g}$ after 50 cycles. The good performance for the carbon micro-particles can be attributed to the smooth surface of the carbon spheres, which is advantageous for the formation of good and efficient passivation layers. This electrode material has promising applications in lithium ion batteries.

Key Words: Carbon micro-particles, Electronic materials, Electrical properties.

\section{INTRODUCTION}

Lithium-ion batteries (LIBs) have been widely used as power sources for portable electronic devices. Their energy density and power density mainly depend on the physical and chemical properties of both cathode and anode materials ${ }^{1}$. Graphite, which has a theoretical maximum capacity of 372 $\mathrm{mAh} / \mathrm{g}$ associated with its maximum $\mathrm{LiC}_{6}$, was commercially used as an anode material for lithium-ion batteries. To meet the increasing demand of lithium-ion batteries with high reversible capacity for electric/hybrid vehicles, many efforts have been made to develop new electrode materials or design novel structures of electrode materials. Recently, graphiterelated materials, especially nano/micro-carbon materials, have received much attention as lithium insertion host materials in lithium-ion batteries basically for their chemical and thermal tolerant, unique structure and electrical conductivity ${ }^{2-4}$. Several carbonaceous materials prepared by carbonization natural organic products, such as sugar, pinecone hull and rice husk have been reported as anode material for lithium-ion batteries ${ }^{5-7}$. However, these carbon anodes usually exhibit poor cycle performance and/or low capacity.

Spheroidal carbons have long been recognized as an interesting material and $\mathrm{Sun}$ and $\mathrm{Li}^{8}$ have reported the synthesis of such material by hydrothermal method using glucose as the carbon source. However, there have only been a few reports dealing with the study of electrochemical performance of the spheroidal carbons for lithium-ion batteries ${ }^{5,9}$. In this paper, we report a facile synthesis of carbon micro particles which have smooth surface and spherical structure with a wide size distribution from $0.5-5.0 \mu \mathrm{m}$. The electrochemical performance of as-obtained carbon micro-particles as an anode material for lithium-ion batteries was investigated. The results showed that carbon micro-particles could exhibit satisfactory electrochemical properties with high capacity and good cycle performance.

\section{EXPERIMENTAL}

Preparation of sample: $2 \mathrm{~g} \beta$-cyclodextrin and $20 \mathrm{~mL}$ distilled water were sealed in a $50 \mathrm{~mL}$ Teflon-lined autoclave and maintained at $160^{\circ} \mathrm{C}$ for $24 \mathrm{~h}$. The product was separated by centrifugation, washed with distilled water for 10 times and dried under vacuum at $80^{\circ} \mathrm{C}$ for $12 \mathrm{~h}$.

The structure of the product was analyzed by X-ray diffraction analysis conducted on a Philips $1730 \mathrm{X}$-ray diffractometer (XRD) with $\mathrm{CuK}_{\alpha}$ radiation. Fourier-transform infrared (FTIR) spectra was recorded on a Nexus 670 FT-IR spectrophotometer. The morphology was examined by a Hitach S4500 field emission scanning electron microscope (FE-SEM).

Electrochemical measurements: The carbon microparticle anode electrode was fabricated by mixing the product as active material with acetylene black and poly(vinylidene fluoride) in weight ratios of 80:10:10, in N-methyl-2pyrrolidone. The resultant slurry was uniformly pasted on $\mathrm{Cu}$ foil with a blade. These prepared electrode sheets were dried at $120^{\circ} \mathrm{C}$ in a vacuum oven for $12 \mathrm{~h}$ and pressed under 
approximately $200 \mathrm{~kg} / \mathrm{cm}^{2}$. CR2032-type coin cells were assembled in an argon-filled glove box for electrochemical measurements. The electrolyte was $1 \mathrm{M} \mathrm{LiPF}_{6}$ in a $1: 1$ mixture of ethylene carbonate and dimethyl carbonate. Li metal foil was used as the counter and reference electrode. A multi-channel battery cycler (LAND2001A) was used for charge-discharge measurements. The voltage range was set between 0.01 and $3.00 \mathrm{~V}$ (versus $\mathrm{Li} / \mathrm{Li}^{+}$) and the current density was $100 \mathrm{~mA} / \mathrm{g}$.

\section{RESULTS AND DISCUSSION}

FTIR spetra of carbon micro-particles is shown in Fig. 1(a). The broad and intense band at $3440 \mathrm{~cm}^{-1}$ and the peak at $1560 \mathrm{~cm}^{-1}$ are possibly attributed to the stretching vibrations and bending vibrations of $\mathrm{O}-\mathrm{H}$ groups, due to the presence of bound water, inhibited water and structural hydroxyl groups. The weak peak located at $1200 \mathrm{~cm}^{-1}$ are most often related to breathing vibrations from C-O groups. Fig. 1(b) shows the XRD pattern of as-synthesized powder. As observed, the positions of the characteristic peaks of the product are consistent with the standard values for the (002) plane of graphite, suggesting that there are no other compounds present in the final product. The sharp peak confirms the high crystallinity of the product.
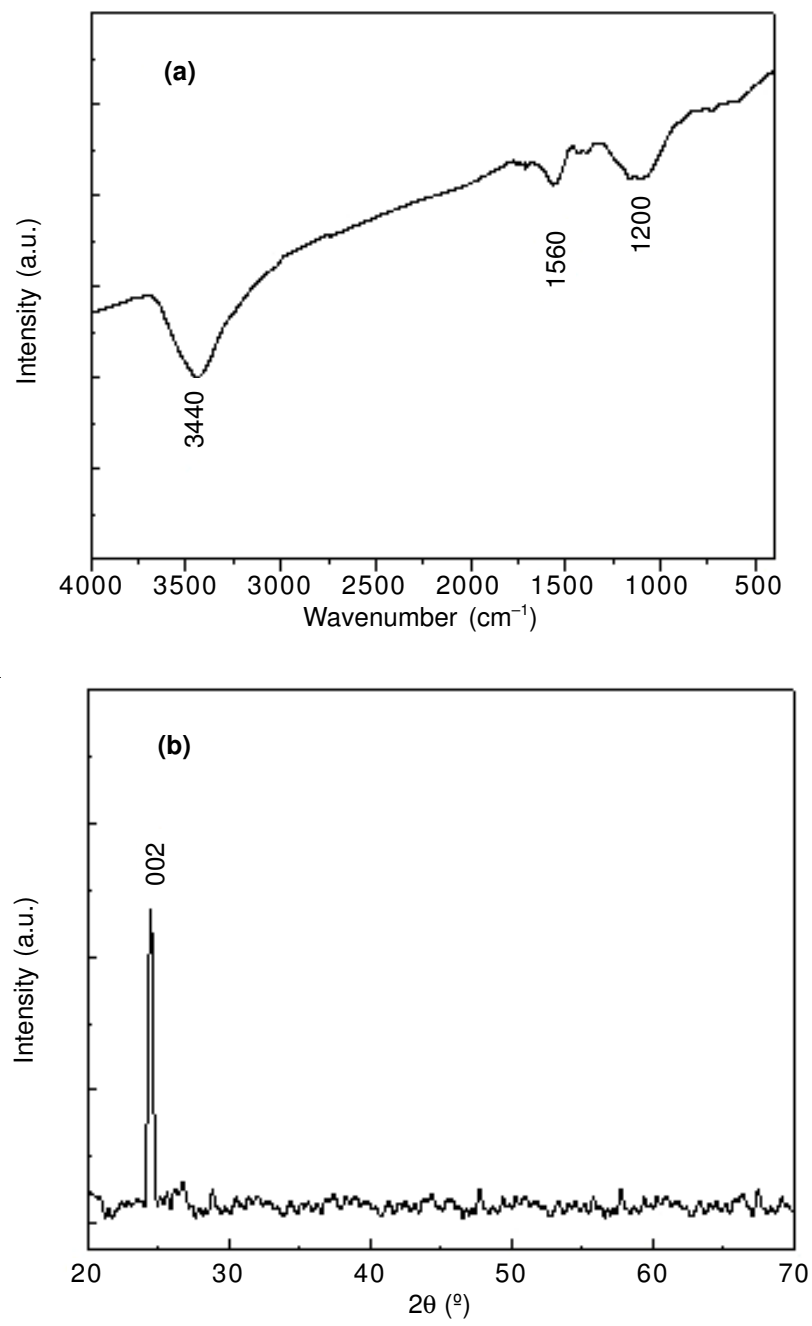

Fig. 1. (a) FT-IR spectra of carbon micro-particles and (b) XRD pattern of carbon micro-particles
Fig. 2 shows a typical SEM image of such carbon microparticles. It can be seen that the carbon particles have wide size distribution in the range from 0.5-5.0 $\mu \mathrm{m}$. The surface of these carbon particles is smooth without any cracks. It is known that surface morphology is an important factor affecting the irreversible capacity. We have examined their suitability as electrode materials for lithium-ion batteries among their diverse potential applications. In the present study, the carbonbased electrode is cathode of $\mathrm{Li} /$ carbon cell because the counter electrode is lithium metal. Li ion intercalation into carbon is the discharge process and the reverse reaction is the charge process. Fig. 3 illustrates the first charge/discharge profile for carbon micro-particles. The initial discharge capacity is as high as $571 \mathrm{mAh} / \mathrm{g}$, while the irreversible capacity is $143 \mathrm{mAh} / \mathrm{g}$. The high irreversible capacity was attributed to the electrolyte decomposition and possible formation of the solid electrolyte interface (SEI). Besides solid electrolyte interface films, the reaction of Li with surface functional groups and irreversible insertion of $\mathrm{Li}$ on the hydrogen terminated edges of graphite sheets also contribute to a fraction of irreversible capacity.

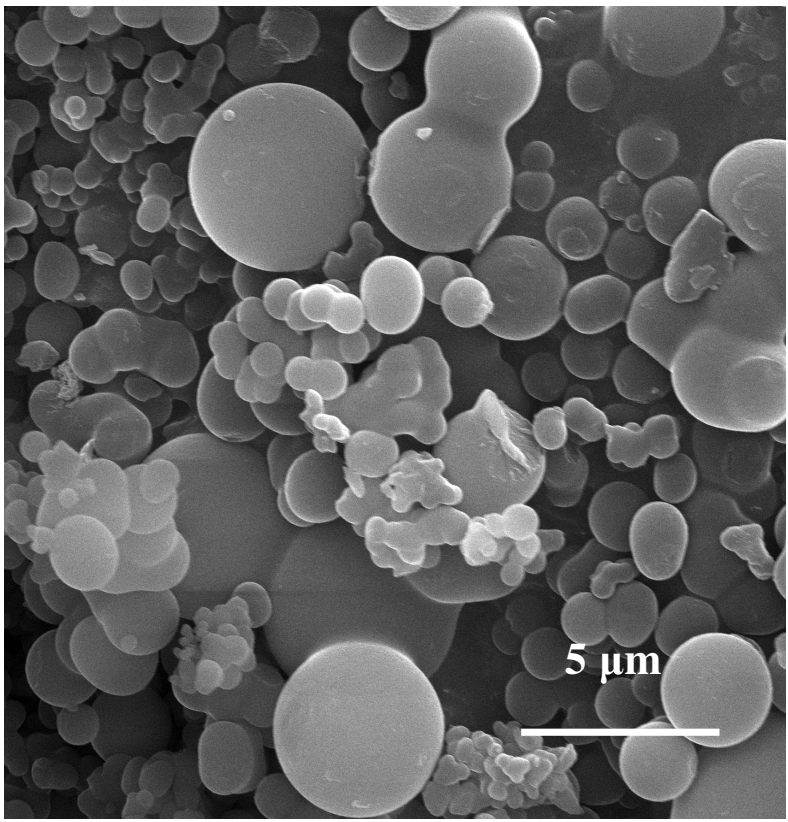

Fig. 2. SEM image of carbon micro-particles

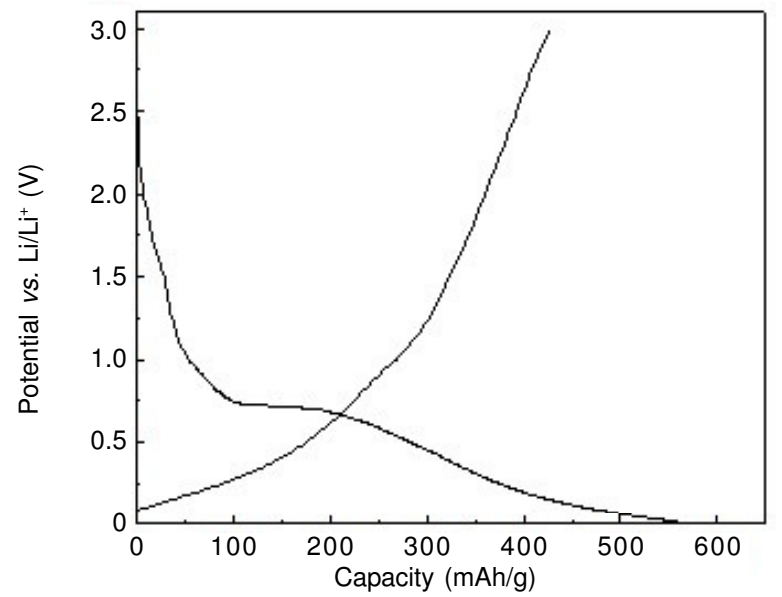

Fig. 3. First charge/discharge profile of carbon micro-particle electrode 
The coulombic efficiency and electrochemical cycle properties of the carbon micro-particle electrode over 50 cycles at the current density of $100 \mathrm{~mA} / \mathrm{g}$ is displayed in Fig. 4. The second and later responses which exhibit stable cyclic properties are important factors when evaluating battery characteristics. As can be seen in Fig. 4, although the first discharge and charge process result in a relative large volume change, which causes anode cracking and pulverization, the capacity drop from the second cycle becomes low. After the first cycle, both discharge and charge capacity fades decrease less and the coulombic efficiency reaches gradually to $97 \%$ and the reversible capacity maintains at above $345 \mathrm{mAh} / \mathrm{g}$. According to the result in Fig. 4, it can be indicated that highly passivated surface films were formed and the structure of carbon micro-particles tends to become stable during the process. These results are consistent with Aurbach et al.'s conclusion, based on the studies of the influence of morphology of graphitic materials on the electrochemical performance of lithium-ion batteries. The conclusion of Aurbach et al. ${ }^{10}$ can be summarized as the smoother the carbon surface, the better the electrochemical performance of carbon in $\mathrm{Li}$ insertion/deinsertion process. The reason is that a smooth surface is advantageous for the formation of good and efficient passivation layers. Such a feature of morphology is expected to be favorable for good cycling stability in the electrode of lithium-ion batteries.

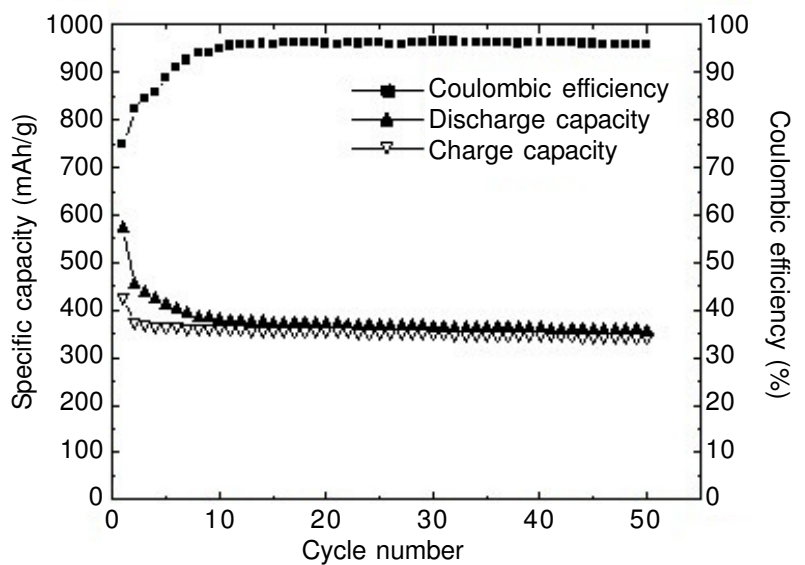

Fig. 4. Cycle performance and coulombic efficiency of carbon microparticle electrode as a function of cycle number

\section{Conclusion}

In summary, carbon micro-particles have been successfully synthesized by a facile hydrothermal method under a relatively mild condition. This sample has a wide size distribution between 0.5 and $5 \mu \mathrm{m}$. Electrochemical performance tests show that the as-synthesized anode exhibits an initial discharge capacity of $571 \mathrm{mAh} / \mathrm{g}$ and a stabilized reversible capacity above $345 \mathrm{mAh} / \mathrm{g}$ after 50 cycles. The good performance for the carbon micro-particles can be attributed to the smooth surface of the carbon spheres, which is advantageous for the formation of good and efficient passivation layers. This electrode material has promising applications in lithium ion batteries because of its high capacity and good cycle performance.

\section{ACKNOWLEDGEMENTS}

This work was supported by the Science and Technology Planned Project of Xi' an City (CXY1134WL36), the Major Scientific Research Fund Project of Xi' an University of Arts and Science (zd201005) and the Research Foundation of Xi'an University of Arts and Science (kyc201011).

\section{REFERENCES}

1. J.M. Tarascon and M. Armand, Nature, 414, 359 (2001)

2. J. Li, C. Wu and L. Guan, J. Phys. Chem. C, 113, 18431 (2009).

3. Y.P. Wu, E. Rahm and R. Holze, J. Power Sources, 114, 228 (2003).

4. H.K. Liu, G.X. Wang, Z.P. Guo, J.Z. Wang and K.J. Konstantinov, J. Nanosci. Nanotechnol., 6, 1 (2006).

5. Z.H. Yi, Y.G. Liang, X.F. Lei, C.W. Wang and J.T. Sun, Mater. Lett., 61, 4199 (2007).

6. Y. Zhang, F. Zhang, G.D. Li and J.S. Chen, Mater. Lett., 61, 5209 (2007).

7. H. Göktepe, H. Sahan and S. Patat, Asian J. Chem., 21, 3186 (2009).

8. X. Sun and Y. Li, Angew. Chem. Int. Ed., 43, 597 (2004).

9. Y.Z. Jin, Y.J. Kim, C. Gao, Y.Q. Zhu, A. Huczko, M. Endo and H.W. Kroto, Carbon, 44, 72472 (2006).

10. D. Aurbach, H. Teller and E. Levi, J. Electrochem. Soc., 149, A1255 (2002) 